\title{
Myocardial Fibrosis as a Predictor of Ventricular Arrhythmias in Patients With Non-ischemic Cardiomyopathy
}

\author{
CĂLIN SCHIAU ${ }^{1}$, DANIEL-CORNELIU LEUCUȚA ${ }^{2}$, SORIN MARIAN DUDEA ${ }^{1}$ and SIMONA MANOLE ${ }^{1}$ \\ ${ }^{1}$ Department of Radiology, “Iuliu Hatieganu” University of Medicine and Pharmacy, Cluj-Napoca, Romania; \\ ${ }^{2}$ Department of Medical Informatics and Biostatistics, \\ "Iuliu Hatieganu” University of Medicine and Pharmacy, Cluj-Napoca, Romania
}

\begin{abstract}
Background/Aim: The aim of the study was to assess the relationship between myocardial fibrosis characteristics (percentage, localization, heterogeneity), evaluated by a non-invasive method such as cardiac magnetic resonance (CMR), with the extrasystolic ventricular arrhythmia in patients with non-ischemic cardiomyopathy. Patients and Methods: The study prospectively included 173 consecutive patients who underwent electrocardiogram Holter monitoring, transthoracic echocardiography and CMR with late gadolinium enhancement (LGE). Results: In univariate analysis, both the presence $(O R=1.05,95 \% C I=1.01-1.09$; $p=0.015)$, the percentage of fibrosis $>15 \%(p=0.018)$, the septum size, the fibrosis in either lateral or septal walls $(p=0.004)$, as well as fibrosis in the midwall $(p=0.019)$ were statistically significant higher in the group with extrasystolic arrhythmia. After adjustment, the percentage of fibrosis $>15 \%$, had higher odds of extra systolic arrhythmia $[O R=3.78$ (95\% CI=1.52-10.62, $p=0.007)]$. Conclusion: The presence, percentage, and localisation of left ventricle myocardial fibrosis characterized by $L G E$ CMR was associated with ventricular arrhythmias.
\end{abstract}

Non-ischemic cardiomyopathy is a common heart muscle disease, without significant coronary artery impairment. The condition is associated with significant morbidity and mortality due to progressive heart failure (HF) and sudden cardiac death $(\mathrm{SCD})(1,2)$.

This article is freely accessible online.

Correspondence to: Daniel Corneliu Leucuta, Department of Medical Informatics and Biostatistics, "Iuliu Hatieganu" University of Medicine and Pharmacy, 6 Pasteur Street, 400349 Cluj-Napoca, Romania. Tel: +40 264597256 int 2502, e-mail: dleucuta@umfcluj.ro

Key Words: CMR, non-ischemic Cardiomyopathy, Sudden cardiac death, Implantable cardioverter-defibrillator.
Implantation of an implantable cardioverter-defibrillator (ICD) has significantly reduced mortality in these patients $(3,4)$, but most of the selected patients according to current guidelines do not really benefit from it (5). At present, the only structural abnormality provided in the practice guidelines for SCD prevention is left ventricular ejection fraction (LVEF), but it is not representative of the pathogenetic substrate, being insensitive and nonspecific (6). Cardiovascular magnetic resonance imaging (CMR) is accepted as the modality of choice for LVEF assessment, being able to classify more accurately patients who benefit from ICD implantation than echocardiography (7).

The arrhythmogenic substrate that produces lethal ventricular arrhythmia and thus SCD, is shown to be myocardial fibrosis, even in the absence of contractile dysfunction (8). It is involved through various mechanisms such as enhanced normal automaticity, abnormal automaticity, triggered activity, and re-entry (9).

The presence and pattern of myocardial fibrosis is highlighted by late gadolinium enhancement (LGE) using CMR, being presently the noninvasive method of choice (10). In patients with no LGE, histopathology confirms the absence or presence of fibrosis (11). The most important elements assessed include LGE by extent, pattern, number of transmural segments (12), existing several patterns and different aspects described depending on the pathogenetic type of cardiomyopathy $(13,14)$. The possibility to characterize the myocardial tissue by LGE adds incremental prognostic value (15).

There are only few studies assessing LGE with ventricular arrhythmia in patients with non-ischemic cardiomyopathy, and many have a limited number of subjects (16). Furthermore, there are is a lack in evidences regarding whether the distribution, location or heterogeneity is predictive of arrhythmias. Finally, the LVEF value as quantified by CMR remains questionable regarding the prediction of arrhythmias, and as a criterion of ICD therapy.

Therefore, the objectives of this study were to assess the relationship between the myocardial fibrosis characteristics 


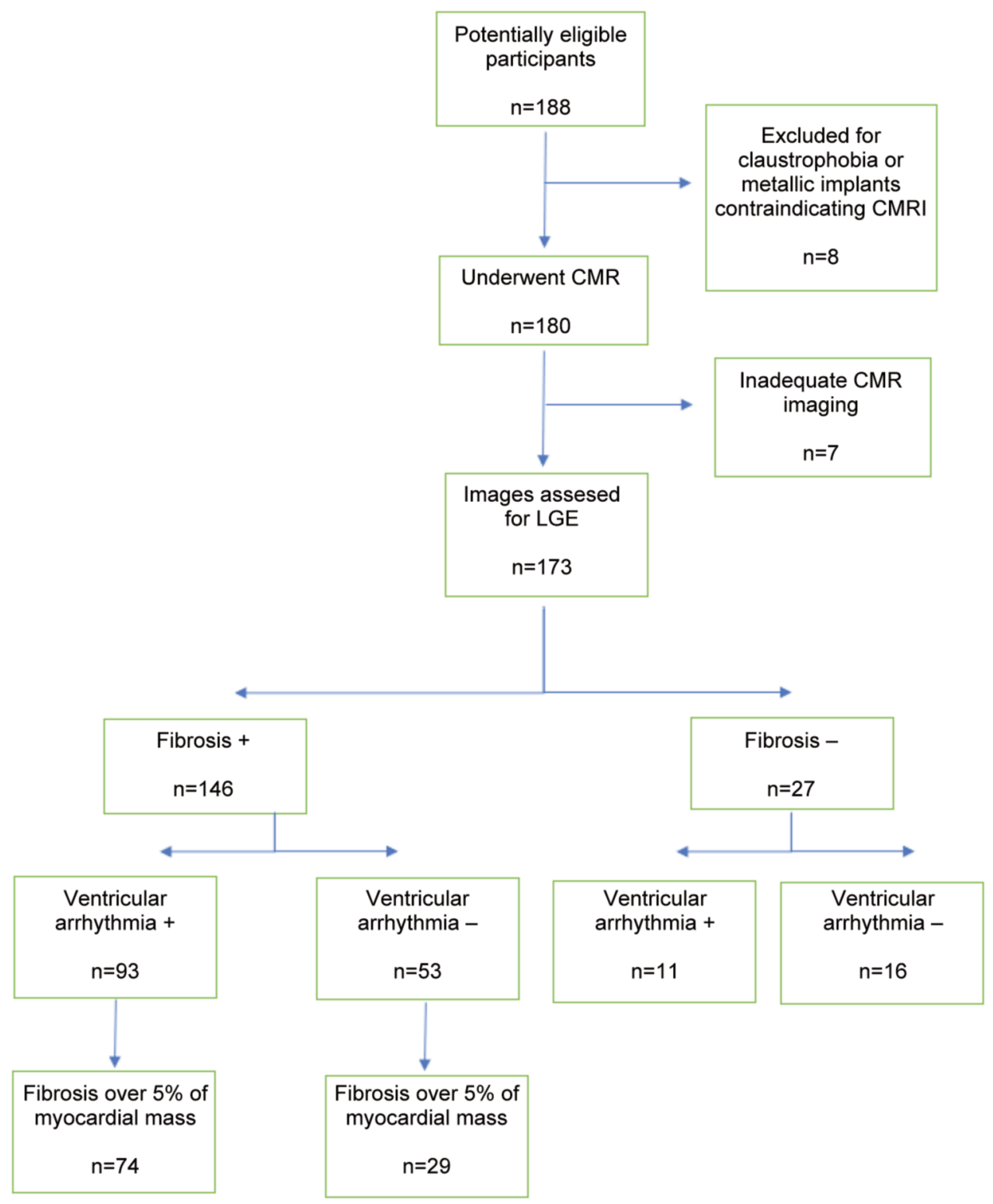

Figure 1. Flowchart showing the study cohort selection, and distribution of study outcomes.

(percentage, localization, heterogeneity), as well as of LVEF observed with the CMR with the extrasystolic ventricular arrhythmia in patients with non-ischemic cardiomyopathy.

\section{Patients and Methods}

Study design and setting. This prospective cohort study included 173 consecutive patients assessed in a regional Cardiology Institute between September 2017 and August 2019. Furthermore, we included patients from the hospital records between August 2016 and September 2017. The study was conducted according to the guidelines of the Declaration of Helsinki, and approved by the
Ethics Committee of "Iuliu Hatieganu" University of Medicine and Pharmacy Cluj-Napoca (protocol code 154/02.04.2018).

Participants. The study included 173 subjects with magnetic resonance imaging (MRI) indication, suffering from cardiomyopathies of nonischemic origin: dilated, hypertrophic and myocardial fibrosis of various non-ischemic etiologies including myocarditis. Inclusion criteria were: dilated cardiomyopathy, hypertrophic cardiomyopathy, myocardial fibrosis of various non-ischemic etiologies including myocarditis, availability of all clinical and lab data sustaining the diagnosis, Holter monitoring, complete cardiac MRI exam.

Subjects were excluded if they suffered from claustrophobia, had a history of a metallic prosthetic implant contraindicating CMR or 


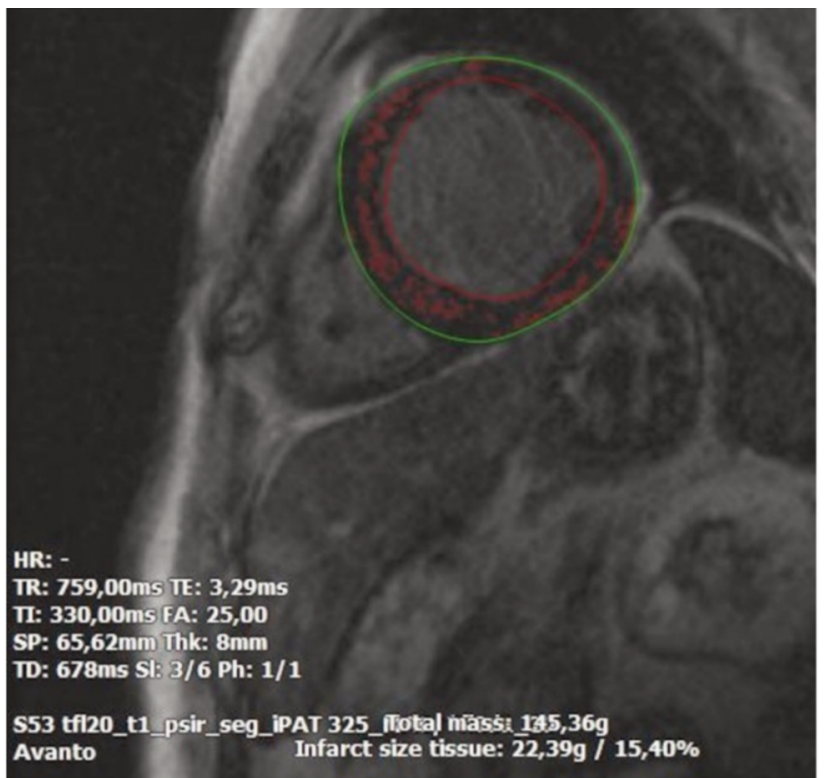

(a)

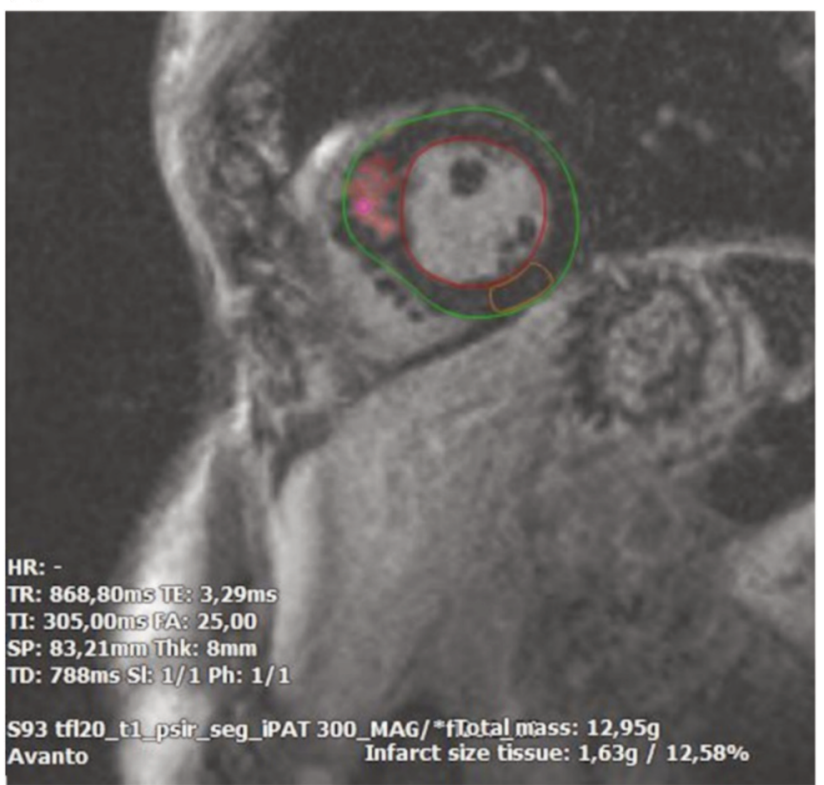

(c)

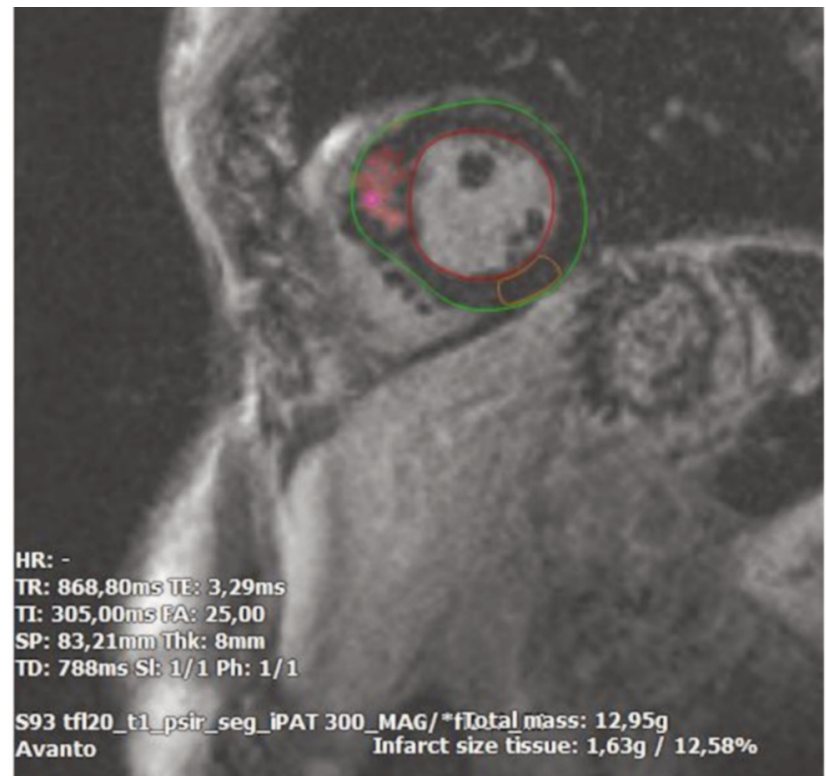

(b)

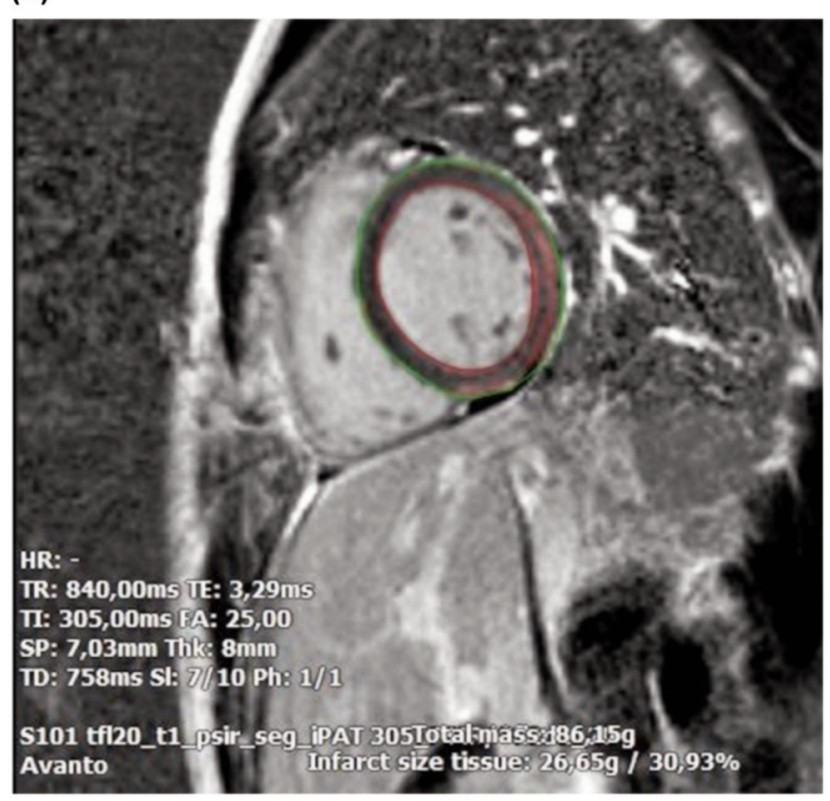

(d)

Figure 2. Example of contour tracing performed on Late Gadolinium Enhancement (LGE) imaging in a patient with (A) dilated cardiomyopathy, (B) hypertrophic cardiomyopathy, and $(C-D)$ myocarditis. Endocardial border is shown in red, epicardial border shown in green, normal reference myocardium shown in an orange frame, a myocardial fibrosis reference contour is shown in pink circle. LGE volume analysis is shown by predefined signal thresholds using full-width-half-width $(F W H W)$ approaches. Myocardial fibrosis in all cases represents more than 5\% of the myocardial mass.

contraindications for contrast administration. In addition, there were several patients with uncontrolled arrhythmias who generated significant acquisition artifacts, the MRI examination not being interpretable. The setting up of the study group is presented in Figure 1.

The patients underwent electrocardiogram (EKG) Holter monitoring, transthoracic echocardiography and CMR examinations during hospitalization, which in most cases did not exceed 7 days. All subjects had complete image acquisition required to evaluate LGE. All of them were enrolled in research protocols after providing written informed consent. The study was carried out with the approval of the University of Medicine and Pharmacy Ethics Commission.

Variables and data sources. The main outcome measures were ventricular extrasystoles and/or ventricular tachycardia, as recorded 
by $24 \mathrm{~h}$ Holter monitoring. The exposure variables were the myocardial fibrosis characteristics (percentage, heterogeneity, localization, transmural extension), as well as of left ventricular ejection fraction, as found by CMR scans. As main predictors and potential confounders, the age, ischemic cardiomyopathy, and heart disease type were assessed.

CMR image acquisition. All subjects underwent CMR scans in a 1.5-T scanner (Avanto MRI system - Siemens Medical Systems, Erlangen, Germany) with an eight-element phased-array surface coil. Retrospective electrocardiographic gating was used during a breathhold of 10 to $15 \mathrm{~s}$ for cardiac volumes and systolic function assessment.

After initial localizer sequences, a stack of steady-state free precession cine images were acquired in the short axis plane from the level of the mitral valve annulus to the left ventricle (LV) apex (Repetition time - TR $3.8 \mathrm{~ms}$, echo time - TE $1.6 \mathrm{~ms}$, 30 phases, slice thickness $8 \mathrm{~mm}$ no gap; flip angle $70^{\circ}$; average in-plane resolution $1.3 \times 1.3 \mathrm{~mm}^{2}$ ). A stack of three long-axis planes (two, three, and four-chamber) was also obtained.

A phase-sensitive inversion recovery sequence for LGE was performed $10 \mathrm{~min}$ after the intravenous administration of $0.1 \mathrm{mmol} / \mathrm{kg}$ of Gadobutrol (Gadovist, Gadavist; Bayer Pharma AG, Leverkusen, Germany) on a stack of LV diastolic short-axis slices with the following parameters: TE $3.17 \mathrm{msec}$; TR $1 \times$ RR interval; flip angle $25^{\circ}$; voxel size $1.9 \times 1.4 \times 8 \mathrm{~mm}^{3}$; and field of view $360 \times 290 \mathrm{~mm}^{2}$.

A scout with increasing inversion time values was performed on a midventricular short-axis slice to determine the inversion time that allowed for optimal nulling of normal myocardium before the LGE images were obtained.

CMR image analysis. Volumetric analysis for quantification of LV volumes and LVEF was based on consecutive short-axis stacks of cine images using QMASS software (Medis Medical Imaging, Leiden, the Netherlands). Papillary muscles were regarded as part of the ventricular cavity. LV volumes and mass were normalized to body surface area.

LGE was assessed by two readers (by a senior and a junior reader) with consensus reading, and was defined as being present only if it was identified in both long-axis and short-axis views. Myocardial fibrosis analysis was performed using QMASS software (Medis Medical Imaging). Endocardial and epicardial LV myocardial borders were manually delineated on the short axis LGE-CMR images (Figure 2).

For LGE volume quantification, the full width at half maximum technique (FWHM) method was used, which takes half of the maximal signal intensity within the myocardial fibrosis as a cutoff for a scarred myocardium. Areas identified as a myocardial fibrosis by the software but not deemed to be myocardial fibrosis by the user were excluded manually.

The FWHM technique for LGE quantification is considered the most reproducible, regardless of underlying etiology, across the spectrum of cardiac disease in which LGE quantification is known to be important. It reduces the required sample sizes by up to onehalf compared to other techniques used in the past (17).

Several complementary aspects of myocardial fibrosis were quantified: presence; heterogeneity; amount of myocardial fibrosis expressed as a percentage of the total LV myocardial mass; affected myocardial segments classified according to the standard American Heart Association 17-segment model; number of transmural affected segments.
Table I. Patient clinical characteristics.

\begin{tabular}{|c|c|c|c|}
\hline $\begin{array}{l}\text { Extrasystolic } \\
\text { ventricular } \\
\text { arrhythmia: }\end{array}$ & $\begin{array}{c}\text { Yes } \\
(\mathrm{n}=104)\end{array}$ & $\begin{array}{c}\text { No } \\
(\mathrm{n}=65)\end{array}$ & $p$-Value \\
\hline $\begin{array}{l}\text { Age (years), } \\
\text { mean (SD) }\end{array}$ & $46.04(16.5)$ & $47.23(15.79)$ & $\begin{array}{c}0.643 \\
{[\mathrm{n} 1=104, \mathrm{n} 2=65]}\end{array}$ \\
\hline Gender $(\mathrm{F})$, no $(\%)$ & $26(24.53)$ & $21(31.34)$ & 0.326 \\
\hline $\begin{array}{l}\mathrm{BMI}\left(\mathrm{kg} / \mathrm{m}^{2}\right) \\
\text { mean }(\mathrm{SD})\end{array}$ & $26.15(5.04)$ & $26.71(4.2)$ & $\begin{array}{c}0.485 \\
{[\mathrm{n} 1=100, \mathrm{n} 2=56]}\end{array}$ \\
\hline Smoker, no $(\%)$ & 47 (46.08) & $21(37.5)$ & 0.298 \\
\hline $\begin{array}{l}\text { Diabetes mellitus, } \\
\text { no }(\%)\end{array}$ & $21(20.59)$ & $11(19.64)$ & 0.888 \\
\hline $\begin{array}{l}\mathrm{SBP}(\mathrm{mmHg}) \\
\text { median }(\mathrm{IQR})\end{array}$ & $120(110-130)$ & $120(110-140)$ & $\begin{array}{c}0.223 \\
{[\mathrm{n} 1=97, \mathrm{n} 2=53]}\end{array}$ \\
\hline $\begin{array}{l}\mathrm{DBP}(\mathrm{mmHg}) \\
\text { median }(\mathrm{IQR})\end{array}$ & $75(62-80)$ & $80(70-80)$ & $\begin{array}{c}0.148 \\
{[\mathrm{n} 1=97, \mathrm{n} 2=53]}\end{array}$ \\
\hline $\begin{array}{l}\text { Associated } \\
\text { cardiovascular } \\
\text { diseases, no }(\%)\end{array}$ & $82(91.11)$ & $41(83.67)$ & 0.189 \\
\hline $\begin{array}{l}\text { Family history } \\
\text { of SCD, no (\%) }\end{array}$ & $7(12.96)$ & $2(7.14)$ & 0.711 \\
\hline Syncope, n (\%) & $4(4.04)$ & $0(0)$ & 0.299 \\
\hline
\end{tabular}

$\mathrm{SD}$, Standard deviation; IQR, interquartile range; BMI, body mass index; SBP, systolic blood pressure; DBP, diastolic blood pressure; SCD, sudden cardiac death.

Statistical analysis. Categorical data were presented as counts and percentages. Normally distributed continuous data were presented as means and standard deviations, while data not following the normal distribution were presented as medians and interquartile ranges. Comparing two groups of categorical data was performed using the chi-squared test or the Fisher exact test (in case of low expected frequencies). Comparing two groups of normally distributed continuous data were performed with the $t$-test for independent samples, while for data not following the normal distribution, the Wilcoxon rank-sum test was used. In order to assess the relationship between the myocardial fibrosis characteristics (percentage, heterogeneity, localization), as well as of LVEF observed with the MRI with the extrasystolic ventricular arrhythmia, and then multivariate logistic regression models for this association were built. The models included known or physiopathological likely implicated characteristics in this relation: age, septal wall involvement, lateral wall involvement, ischemic cardiomyopathy, heart disease type. Next, since the dataset contained some missing values, an iterative imputation method based on random forests (18) was used for the variables included in the regression. Then the main multivariate model (model 1: age (years), myocardial fibrosis $>15 \%$ (26), myocardial fibrosis heterogeneity, septal wall fibrosis, lateral wall fibrosis, ischemic cardiomyopathy, LVEF MRI) was fitted again on these data. The main multivariate model (model 1) was specified to have no more than 7 degrees of freedom - when predicting the extra systolic ventricular arrhythmia, to prevent overfitting. For all the models, the goodness-of-fit with the Hosmer and Lemeshow test was checked. For continuous predictors, the assumption of linearity to the logit using splines was checked, and it was found that the percentage of myocardial fibrosis assumption did not hold; thus, was 
Table II. Heart-related characteristics comparison between extrasystolic ventricular arrhythmia.

\begin{tabular}{|c|c|c|c|}
\hline Extrasystolic ventricular arrhythmia: & $\begin{array}{c}\text { Yes } \\
(\mathrm{n}=106)\end{array}$ & $\begin{array}{c}\text { No } \\
(\mathrm{n}=67)\end{array}$ & $p$-Value \\
\hline NYHA class, n (\%) & $\begin{array}{c}1: 26(27.96) \\
2: 27(29.03) \\
3: 39(41.94) \\
4: 1(1.08)\end{array}$ & $\begin{array}{c}\text { 1: } 18(36) \\
2: 14(28) \\
3: 18(36) \\
4: 0(0)\end{array}$ & 0.751 \\
\hline Ventricular tachycardia, n (\%) & $48(45.28)$ & $9(13.43)$ & $<0.001$ \\
\hline Interventricular septal diameter, median (IQR) & $10(8-11)$ & $11(10-13)$ & $<0.001$ \\
\hline LV Lateral wall diameter, median (IQR) & $7.75(6-10)$ & $9(6.25-11)$ & 0.347 \\
\hline Myocardial fibrosis mass (g), median (IQR) & $16.56(5.97-26.6)$ & $7.32(0-16.64)$ & $<0.001$ \\
\hline Myocardial fibrosis (\%), median (IQR) & $11.72(5.26-17.18)$ & $7.42(0-12.46)$ & 0.002 \\
\hline Myocardial fibrosis, $\mathrm{n}(\%)$ & $95(89.62)$ & $51(76.12)$ & 0.017 \\
\hline Myocardial fibrosis $>5 \%, \mathrm{n}(\%)$ & $75(76.53)$ & $28(54.9)$ & 0.007 \\
\hline Myocardial fibrosis $>15 \%, \mathrm{n}(\%)$ & $30(30.61)$ & $7(13.73)$ & 0.024 \\
\hline Myocardial fibrosis heterogeneity, n (\%) & $63(64.95)$ & $28(56)$ & 0.29 \\
\hline Basal, n (\%) & $76(73.08)$ & $40(61.54)$ & 0.116 \\
\hline Mid, n (\%) & $80(76.92)$ & $39(60)$ & 0.019 \\
\hline Apical, n $(\%)$ & $50(48.08)$ & $33(50.77)$ & 0.733 \\
\hline Lateral wall or septum involvement, $\mathrm{n}(\%)$ & $92(86.79)$ & $46(68.66)$ & 0.004 \\
\hline
\end{tabular}

NYHA, New York Hearth Association; LV, left ventricle; IQR, interquartile range.

used the percentage of myocardial fibrosis $>15 \%$ instead (26). For the multivariable logistic regression models, the multicollinearity and the functional form were checked. For all models, the odds ratio, along with the $95 \%$ confidence interval and the $p$-value were reported. For all statistical tests used, a significance level alpha of 0.05 was chosen, and the two-tailed $p$-value was computed. All statistical analyses were carried out with the $\mathrm{R}$ environment for statistical computing and graphics (R Foundation for Statistical Computing, Vienna, Austria), version 4.0.2 (19).

\section{Results}

The study sample consisted of 173 patients diagnosed with dilated cardiomyopathy, hypertrophic cardiomyopathy, myocarditis. The mean age of patients was 46.5 years ( $\mathrm{SD}=16.2$ years), ranging from 9 to 81 years old. The majority of patients were males $(n=126)(72.8 \%)$. There were no statistically significant differences between patients with and without extrasystolic arrhythmia regarding the main clinical characteristics (Table I).

Regarding hearth pathology, the cardiomyopathy type, and the New York Heart Association (NYHA) class were similar between patients with and without extrasystolic arrhythmia (Table II). Similarly, the heart weight and the left ventricle wall-size were not statistically significantly different between the two groups. However, the septum size was statistically significant larger in the group without extrasystolic arrhythmia. The mass and percentage of fibrosis were statistically significant higher in the group with extrasystolic arrhythmia. Other findings were that fibrosis in either lateral or septal walls, and fibrosis in the midwall were statistically significant more frequent in the group with extrasystolic ventricular arrhythmia.

In order to characterize the relationship between myocardial fibrosis characteristics (percentage, heterogeneity, localization), as well as of LVEF observed with the MRI with the extrasystolic ventricular arrhythmia, several logistic regression models were built (Table III). An increased percentage of myocardial fibrosis was statistically significantly associated with extrasystolic ventricular arrhythmia episodes in the univariate model. Furthermore, a model (model 1) was built including age (years), myocardial fibrosis $>15 \%$, myocardial fibrosis heterogeneity, septal wall fibrosis, lateral wall fibrosis, ischemic cardiomyopathy, LVEF MRI variables. In this model the relation between of myocardial fibrosis and extrasystolic ventricular arrhythmia episodes, was close to the statistical significance level, as well as in a model that included the hearth disease types. Finally, model 1 was fitted on the same dataset, but with missing values imputed. Here too, an increased percentage of myocardial fibrosis was found, being statistically significant associated with extrasystolic ventricular arrhythmia episodes. However, in all models the myocardial fibrosis heterogeneity, the fibrosis localization (septal wall, or lateral wall), as well as LVEF MRI were not statistically significant associated with the extrasystolic ventricular arrhythmia episodes. There was no statistically significant relation between the hearth disease type and the extrasystolic ventricular arrhythmia episodes in the univariate and multivariate models (where it was used to control for confounders). Model 1 was computed on 173 observations. The Hosmer Lemeshow goodness of fit test $p$-value was 0.341 . For 


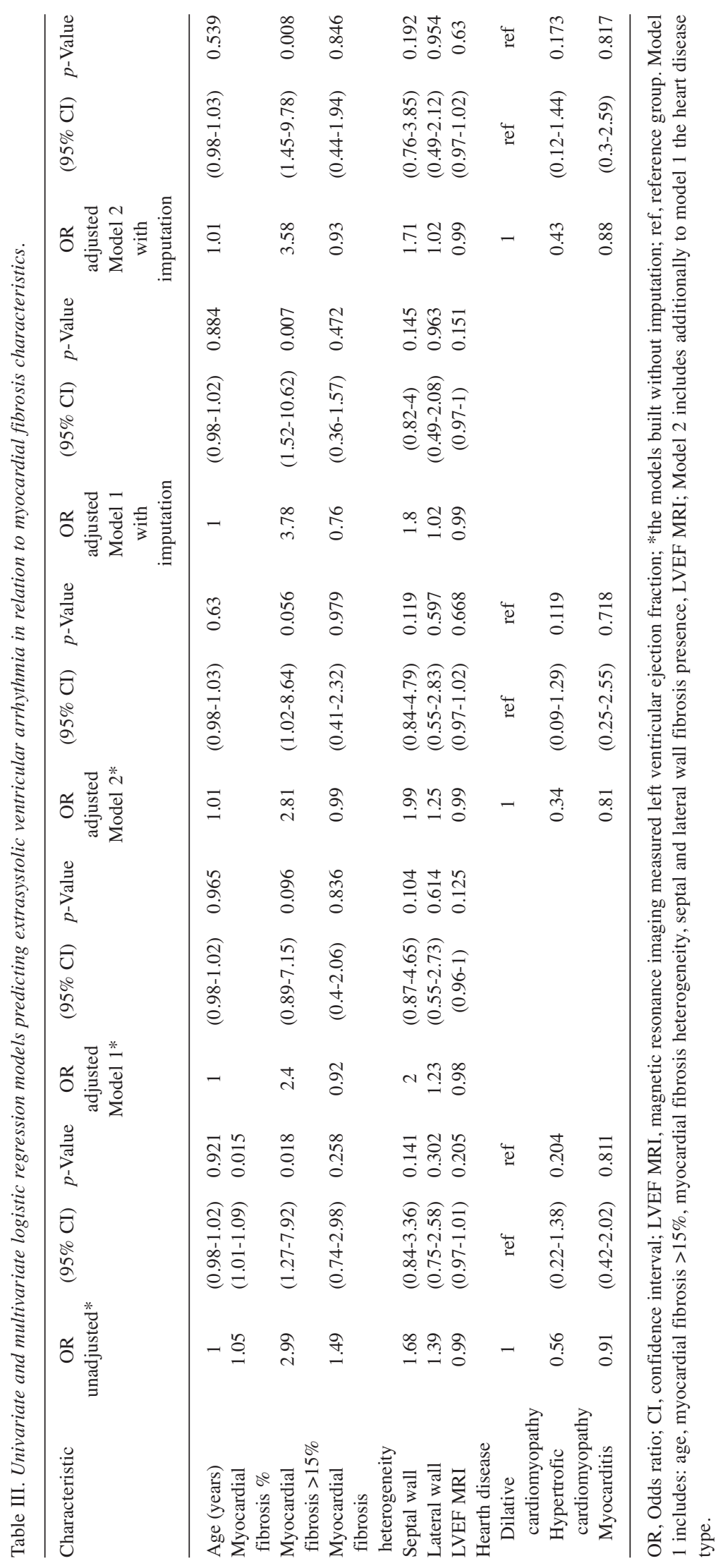


this model, the overall percent of correct classification was $66.5 \%$, and the area under the receiver operating characteristic was $69.2(61.1-77.2)$

\section{Discussion}

The present study provides further support for the predictive utility of LGE quantification in patients with non-ischemic cardiomyopathy (NICM). The study succeeded to objectify that: i) myocardial fibrosis was a common feature in NICM; ii) regardless of cardiomyopathy etiology, there was an association between ventricular arrhythmias and the presence, percentage (threshold value of $15 \%$ of myocardial mass), and specific location of fibrosis; iii) a significant association between LVEF or and ventricular arrhythmias was not observed. Likewise, no significant association was observed with the NYHA class.

LGE assessment using CMR is considered the noninvasive method of choice for imaging the presence and pattern of myocardial fibrosis (10). We found that both the presence and extent of myocardial fibrosis were associated with an increased likelihood of ventricular arrhythmias. A significant association from a threshold value of $15 \%$ of myocardial mass was observed. This association was independent of LVEF, NYHA class and other established prognostic factors. Our findings suggest that detection and quantification of midwall fibrosis by LGE-CMR may represent useful markers for the risk stratification of ventricular arrhythmia, and possibly (further studies needed) HF and death for patients with non-ischemic cardiomyopathy. There are other studies that support this aspect, where a relation between LGE transmurality and an increased risk for arrhythmic events was also found $(20,21)$. Regarding the subgroup of patients with hypertrophic cardiomyopathy (HCM), there are two independent studies that have consistently found that a large amount of LGE predicts major adverse cardiac events in patients with hypertrophic cardiomyopathy $(22,23)$, a threshold value of $15 \%$ being suggested by Chan et al. (24).

The present study observed that fibrosis in either lateral or septal walls, as well as fibrosis in the midwall are statistically significant more frequent in the group with extrasystolic ventricular arrhythmia. The midwall localization was also observed in the study carried out by Gulati et al., where patients with chronic dilated NICM had an increased frequency of a secondary composite arrhythmic end point consisting of SCD or aborted SCD (21).

In this study a possible link between extrasystolic ventricular arrhythmia and LVEF was analyzed. No association was found, irrespective of NICM etiology, even though MRI examination is considered the gold standard in its calculation because of its high accuracy and reproducibility (25). The same was observed by analyzing the NYHA class. As in the present study, the studies performed by Almehmadi and Gulati observed that LVEF was not associated with arrhythmic events in dilated cardiomyopathy suggesting that the prognostic value of LGE by CMR might be superior and independent from $\operatorname{LVEF}(21,26)$.

Myocardial fibrosis assessed by CMR can ameliorate clinical practice regarding therapeutic decisions in nonischemic cardiomyopathy patients. The significant link between myocardial fibrosis and arrhythmias suggests an increased risk of sudden cardiac death through malign arrhythmias. This SCD can be prevented with ICD implantation. Therefore, assessing myocardial fibrosis by CMR, can be an important decision criterion in deciding ICD implantation. Current clinical guidelines recommend ICD implantation in patients with advanced HF using LVEF as the main criterion. But, it was shown that the majority of patients never receive appropriate therapy (5). Therefore, selecting patients for ICD therapy based on LVEF alone, is an imperfect and inexact method. The present study observed a significant link between myocardial fibrosis and arrhythmias, and no significant association between LVEF was measured by the reference method (CMR) or the NYHA class. Therefore, CMR could add incremental prognostic value, with its precise tissue characterization, leading to better classifying patients who benefit from ICD implantation, and in determining their treatment with ICD. One possibility could be the replacement of LVEF criterion altogether, with myocardial fibrosis assessed by CMR, in the choice of ICD implantation. Another possibility could be the use of CMR as a supplementary criterion along LVEF. On one hand, patients who fall outside current indications could benefit from ICD, and have their life expectancy improved. Thus, in asymptomatic patients with normal LVEF, a high myocardial fibrosis would help the decision to introduce ICD therapy. A reduced myocardial fibrosis would reassure that there is no need for ICD therapy. On the other hand, in patients with low LVEF, the absence of myocardial fibrosis might avoid ICD implantation due to low risk of arrhythmias, and low risk of SCD. This might bring major benefits due to the limitation of exposure to specific complications of the device implantation, and lower costs, in patients who have a low risk of SCD (where ICD therapy would have a reduced benefit, without a significant improvement in life expectancy). Another important fact is that, according to Chen et al., the absence of focal myocardial fibrosis (as our study quantified too) is associated with better response to LV reverse modeling following cardiac resynchronization therapy (CRT) (27). Also, there is a lower rate of arrhythmic events observed in responders to CRT (28). Thus, if there are patients without myocardial fibrosis, with high prognosis for LV remodeling, it would be more appropriate to install a pacemaker and not a defibrillator. Taking all this into account myocardial fibrosis assessed by CMR might have an important role on ameliorating clinical practice regarding complex decisions in the use of ICD therapy or CRT, and also in its cost-effectiveness. 
The strengths of our study are represented by its number of subjects, which is higher than that of many other studies carried out on this topic. Also, myocardial fibrosis was evaluated quantitatively. Moreover, besides assessing the fibrosis degree relation with arrhythmias, it also checked the location and heterogeneity of fibrosis, which were found to be important.

Regarding limitations, the diffuse myocardial fibrosis has not been analyzed, through techniques such as T1 mapping and extracellular volume fraction calculation. LGE only assesses fibrosis distributed in a regional pattern. Diffuse myocardial fibrosis is also likely to influence the development of ventricular arrhythmias. A limitation of the MRI technique is its specific absolute and relative contraindications or related to contrast injection. In addition, there were several patients with significant arrhythmias who generated significant acquisition artifacts, the MRI examination not being interpretable. Since this study is observational in nature, residual confounding cannot be ruled out. To minimize this bias, we adjusted the regression for known important confounders.

\section{Conclusion}

The present study showed that above $15 \%$ fibrosis of the total myocardial mass was significantly correlated with malignant arrhythmias. This observation suggests that a new criterion for ICD therapy could be the percentage of fibrosis from the total myocardial mass. Other findings were that fibrosis in either lateral or septal walls, as well as fibrosis in the midwall were statistically significant more frequent in the group with extrasystolic ventricular arrhythmia in univariate analyses.

The present study did not find any statistical relationship between LVEF and the frequency of arrhythmic events. This suggest that LVEF might not be an optimal predictor of arrhythmic events in NICM, even though it is an important criterion in the introduction of ICD therapy. Therefore, the prognostic value of fibrosis as LGE quantified by CMR was superior and independent from LVEF and could be a powerful tool to improve risk stratification in patients irrespective of NICM etiology.

\section{Conflicts of Interest}

The Authors declare no conflicts of interest.

\section{Authors' Contributions}

Conceptualization, C.S., S.M.D. and S.M.; methodology, C.S., D.C.L., S.M.D. and S.M.; software, S.M.; validation, C.S. and S.M.; formal analysis, C.S., D.C.L., S.M.D. and S.M.; investigation, C.S.; resources, S.M.D. and S.M.; data curation, C.S., D.C.L. and S.M.; writingoriginal draft preparation, C.S.; writing-review and editing, C.S.,
D.C.L., S.M.D. and S.M.; visualization, C.S.; supervision, S.M.D. and S.M.; project administration, S.M.D. and S.M. The Authors had full access to the data and take responsibility for its integrity. All Authors have read and agreed to the manuscript as written.

\section{References}

1 Al-Khatib SM, Stevenson WG, Ackerman MJ, Bryant WJ, Callans DJ, Curtis AB, Deal BJ, Dickfeld T, Field ME, Fonarow GC, Gillis AM, Granger CB, Hammill SC, Hlatky MA, Joglar JA, Kay GN, Matlock DD, Myerburg RJ and Page RL: 2017 AHA/ACC/HRS guideline for management of patients with ventricular arrhythmias and the prevention of sudden cardiac death: A report of the American College of Cardiology/American Heart Association task force on clinical practice guidelines and the Heart Rhythm Society. Circulation 138(13): e272-e391, 2018. PMID: 29084731. DOI: 10.1161/CIR.0000000000000549

2 Schiau C, Schiau Ş, Dudea SM and Manole S: Cardiovascular magnetic resonance: Contribution to the exploration of cardiomyopathies. Med Pharm Rep 92(4): 326-336, 2019. PMID: 31750431. DOI: $10.15386 / \mathrm{mpr}-1343$

3 Ip J, Kantipudi S, Khasnis A, Virk IS and Khan S Prophylactic defibrillator implantation in patients with non-ischemic dilated cardiomyopathy. Congest Heart Fail 10(5) 257-258, 2004. DOI 10.1111j.1527-5299.2004.02811.x

4 Bardy GH, Lee KL, Mark DB, Poole JE, Packer DL, Boineau R, Domanski M, Troutman C, Anderson J, Johnson G, McNulty SE, Clapp-Channing N, Davidson-Ray LD, Fraulo ES, Fishbein DP, Luceri RM, Ip JH and Sudden Cardiac Death in Heart Failure Trial (SCD-HeFT) Investigators.: Amiodarone or an implantable cardioverter-defibrillator for congestive heart failure. N Engl J Med 352(3): 225-237, 2005. PMID: 15659722. DOI: 10.1056/NEJMoa043399

5 Ezekowitz JA, Rowe BH, Dryden DM, Hooton N, Vandermeer $\mathrm{B}$, Spooner C and McAlister FA: Systematic review: Implantable cardioverter defibrillators for adults with left ventricular systolic dysfunction. Ann Intern Med 147(4): 251-262, 2007. PMID: 17709759. DOI: 10.7326/0003-4819-147-4-200708210-00007

6 Wellens HJ, Schwartz PJ, Lindemans FW, Buxton AE, Goldberger JJ, Hohnloser SH, Huikuri HV, Kääb S, La Rovere MT, Malik M, Myerburg RJ, Simoons ML, Swedberg K, Tijssen J, Voors AA and Wilde AA: Risk stratification for sudden cardiac death: Current status and challenges for the future. Eur Heart J 35(25): 1642-1651, 2014. PMID: 24801071. DOI: 10.1093/ eurheartj/ehu176

7 Wu KC and Calkins H: Powerlessness of a number: Why left ventricular ejection fraction matters less for sudden cardiac death risk assessment. Circ Cardiovasc Imaging 9(10): 2016. PMID: 27729366. DOI: 10.1161/CIRCIMAGING.116.005519

8 Wu KC: Sudden cardiac death substrate imaged by magnetic resonance imaging: From investigational tool to clinical applications. Circ Cardiovasc Imaging 10(7): 2017. PMID: 28637807. DOI: 10.1161/CIRCIMAGING.116.005461

9 Cherry EM, Fenton FH and Gilmour RF Jr: Mechanisms of ventricular arrhythmias: A dynamical systems-based perspective. Am J Physiol Heart Circ Physiol 302(12): H2451-H2463, 2012. PMID: 22467299. DOI: 10.1152/ajpheart.00770.2011

10 Simonetti OP, Kim RJ, Fieno DS, Hillenbrand HB, Wu E, Bundy JM, Finn JP and Judd RM: An improved MR imaging technique 
for the visualization of myocardial infarction. Radiology 218(1): 215-223, 2001. PMID: 11152805. DOI: 10.1148/radiology. 218.1.r01ja50215

11 Iles LM, Ellims AH, Llewellyn H, Hare JL, Kaye DM, McLean CA and Taylor AJ: Histological validation of cardiac magnetic resonance analysis of regional and diffuse interstitial myocardial fibrosis. Eur Heart J Cardiovasc Imaging 16(1): 14-22, 2015. PMID: 25354866. DOI: 10.1093/ehjci/jeu182

12 Gräni C, Benz DC, Gupta S, Windecker S and Kwong RY: Sudden cardiac death in ischemic heart disease: From imaging arrhythmogenic substrate to guiding therapies. JACC Cardiovasc Imaging 13(10): 2223-2238, 2020. PMID: 31864982. DOI: 10.1016/j.jcmg.2019.10.021

13 Klem I, Weinsaft JW, Bahnson TD, Hegland D, Kim HW, Hayes B, Parker MA, Judd RM and Kim RJ: Assessment of myocardial scarring improves risk stratification in patients evaluated for cardiac defibrillator implantation. J Am Coll Cardiol 60(5): 408420, 2012. PMID: 22835669. DOI: 10.1016/j.jacc.2012.02.070

14 Maron MS: Clinical utility of cardiovascular magnetic resonance in hypertrophic cardiomyopathy. J Cardiovasc Magn Reson 14: 13, 2012. PMID: 22296938. DOI: 10.1186/1532-429X-14-13

15 Pontone G, Guaricci AI, Andreini D, Solbiati A, Guglielmo M, Mushtaq S, Baggiano A, Beltrama V, Fusini L, Rota C, Segurini C, Conte E, Gripari P, Dello Russo A, Moltrasio M, Tundo F, Lombardi F, Muscogiuri G, Lorenzoni V, Tondo C, Agostoni P, Bartorelli AL and Pepi M: Prognostic benefit of cardiac magnetic resonance over transthoracic echocardiography for the assessment of ischemic and nonischemic dilated cardiomyopathy patients referred for the evaluation of primary prevention implantable cardioverter-defibrillator therapy. Circ Cardiovasc Imaging 9(10): 2016. PMID: 27729359. DOI: 10.1161/ CIRCIMAGING.115.004956

16 Disertori M, Rigoni M, Pace N, Casolo G, Masè M, Gonzini L, Lucci D, Nollo G and Ravelli F: myocardial fibrosis assessment by LGE Is a powerful predictor of ventricular tachyarrhythmias in ischemic and nonischemic LV dysfunction: A meta-analysis. JACC Cardiovasc Imaging 9(9): 1046-1055, 2016. PMID: 27450871. DOI: 10.1016/j.jcmg.2016.01.033

17 Flett AS, Hasleton J, Cook C, Hausenloy D, Quarta G, Ariti C, Muthurangu V and Moon JC: Evaluation of techniques for the quantification of myocardial scar of differing etiology using cardiac magnetic resonance. JACC Cardiovasc Imaging 4(2): 150156, 2011. PMID: 21329899. DOI: 10.1016/j.jcmg.2010.11.015

18 Stekhoven DJ and Bühlmann P: MissForest-non-parametric missing value imputation for mixed-type data. Bioinformatics 28(1): 112118, 2012. PMID: 22039212. DOI: 10.1093/bioinformatics/btr597

$19 \mathrm{R}$ core team: R: A language and environment for statistical computing. Vienna, Austria R Foundation for Statistical Computing, 2020.

20 Masci PG, Doulaptsis C, Bertella E, Del Torto A, Symons R, Pontone G, Barison A, Droogné W, Andreini D, Lorenzoni V, Gripari P, Mushtaq S, Emdin M, Bogaert J and Lombardi M: Incremental prognostic value of myocardial fibrosis in patients with non-ischemic cardiomyopathy without congestive heart failure. Circ Heart Fail 7(3): 448-456, 2014. PMID: 24647118. DOI: 10.1161/CIRCHEARTFAILURE.113.000996

21 Gulati A, Jabbour A, Ismail TF, Guha K, Khwaja J, Raza S, Morarji K, Brown TD, Ismail NA, Dweck MR, Di Pietro E, Roughton M, Wage R, Daryani Y, O'Hanlon R, Sheppard MN, Alpendurada F, Lyon AR, Cook SA, Cowie MR, Assomull RG,
Pennell DJ and Prasad SK: Association of fibrosis with mortality and sudden cardiac death in patients with nonischemic dilated cardiomyopathy. JAMA 309(9): 896-908, 2013. PMID: 23462786. DOI: 10.1001/jama.2013.1363

22 Bruder O, Wagner A, Jensen CJ, Schneider S, Ong P, Kispert EM, Nassenstein K, Schlosser T, Sabin GV, Sechtem U and Mahrholdt H: Myocardial scar visualized by cardiovascular magnetic resonance imaging predicts major adverse events in patients with hypertrophic cardiomyopathy. J Am Coll Cardiol 56(11): 875-887, 2010. PMID: 20667520. DOI: 10.1016/j.jacc. 2010.05.007

23 Green JJ, Berger JS, Kramer CM and Salerno M: Prognostic value of late gadolinium enhancement in clinical outcomes for hypertrophic cardiomyopathy. JACC Cardiovasc Imaging 5(4): 370377, 2012. PMID: 22498326. DOI: 10.1016/j.jcmg.2011.11.021

24 Chan RH, Maron BJ, Olivotto I, Pencina MJ, Assenza GE, Haas T, Lesser JR, Gruner C, Crean AM, Rakowski H, Udelson JE, Rowin E, Lombardi M, Cecchi F, Tomberli B, Spirito P, Formisano F, Biagini E, Rapezzi C, De Cecco CN, Autore C, Cook EF, Hong SN, Gibson CM, Manning WJ, Appelbaum E and Maron MS: Prognostic value of quantitative contrastenhanced cardiovascular magnetic resonance for the evaluation of sudden death risk in patients with hypertrophic cardiomyopathy. Circulation 130(6): 484-495, 2014. PMID: 25092278. DOI: 10.1161/CIRCULATIONAHA.113.007094

25 Grothues F, Smith GC, Moon JC, Bellenger NG, Collins P, Klein HU and Pennell DJ: Comparison of interstudy reproducibility of cardiovascular magnetic resonance with two-dimensional echocardiography in normal subjects and in patients with heart failure or left ventricular hypertrophy. Am J Cardiol 90(1): 29-34, 2002. PMID: 12088775. DOI: 10.1016/s0002-9149(02)02381-0

26 Almehmadi F, Joncas SX, Nevis I, Zahrani M, Bokhari M, Stirrat J, Fine NM, Yee R and White JA: Prevalence of myocardial fibrosis patterns in patients with systolic dysfunction: Prognostic significance for the prediction of sudden cardiac arrest or appropriate implantable cardiac defibrillator therapy. Circ Cardiovasc Imaging 7(4): 593-600, 2014. PMID: 24902587. DOI: 10.1161/CIRCIMAGING.113.001768

27 Chen Z, Sohal M, Sammut E, Child N, Jackson T, Claridge S, Cooklin M, O’Neill M, Wright M, Gill J, Chiribiri A, Schaeffter T, Carr-White G, Razavi R and Rinaldi CA: Focal but not diffuse myocardial fibrosis burden quantification using cardiac magnetic resonance imaging predicts left ventricular reverse modeling following cardiac resynchronization therapy. $\mathrm{J}$ Cardiovasc Electrophysiol 27(2): 203-209, 2016. PMID: 26463874. DOI: $10.1111 /$ jce. 12855

28 Ruwald MH, Solomon SD, Foster E, Kutyifa V, Ruwald AC, Sherazi S, McNitt S, Jons C, Moss AJ and Zareba W: Left ventricular ejection fraction normalization in cardiac resynchronization therapy and risk of ventricular arrhythmias and clinical outcomes: Results from the multicenter automatic defibrillator implantation trial with cardiac resynchronization therapy (MADIT-CRT) trial. Circulation 130(25): 2278-2286, 2014. PMID: 25301831. DOI: 10.1161/CIRCULATIONAHA.114.011283

Received January 24, 2021

Revised February 10, 2021

Accepted February 18, 2021 